\title{
SISTEMA DE TIEMPO-MODO-ASPECTO EN CRIOLLOS DE BASE ESPAÑOLA
}

\author{
Carla Victoria Jara M.
}

\begin{abstract}
RESUMEN
En este trabajo se comparan los sistemas de tiempo, modo y aspecto de tres criollos de base española. La tesis de Derek Bickerton, de que las lenguas criollas comparten de manera universal un sistema particular de TMA, se evalúa con los criollos incluidos en este estudio, el Palenquero (Colombia), Papiamento (Antillas Neerlandesas) y los criollos de base española desarrollados en Filipinas.
\end{abstract}

\begin{abstract}
In this paper three Spanish-based creoles are compared in regard to their tense-mood-aspect (TMA) systems. The thesis set forth by Derek Bickerton, that a particular TMA system is a universally shared feature of creole languages, is assessed with respect to the creoles included in this study, i.e. Palenquero (Colombia), Papiamento (Netherlands Antilles) and the Spanish-based creoles developed in the Phillipines.
\end{abstract}

\section{Introducción}

\subsection{Propósito}

El propósito de este estudio es comparar los sistemas de tiempo-modo-aspecto de lenguas criollas que comparten el mismo superestrato, español, pero difieren en sus respectivos substratos, ya que se han desarrollado en diferentes regiones del mundo. En lo sucesivo, me referiré a estos sistemas de tiempo-modo-aspecto de las gramáticas de lenguas criollas abreviadamente como sistema de TMA. Los criollos comparados son: el palenquero del norte de Colombia, el papiamento de las Antillas Holandesas, y los criollos de base española de las Filipinas: ermitaño, caviteño y zamboagueño. Me adscribo aquí a la tesis de que el papiamento es de base española y no portuguesa, de acuerdo con los argumentos expuestos en Ferrol (1982). Reconozco sin embargo la condición polémica de esta teoría, refutada por quienes defienden la base portuguesa del papiamento (cp. Yang 1988).

La codificación de las categorías verbales ha sido un componente fundamental en la caracterización de la gramática de lenguas criollas. Bickerton (1979) afirma que un sistema 
particular de tiempo-modo-aspecto (TMA) es un rasgo compartido por todos los criollos existentes. Mi propósito es determinar hasta dónde se cumple esta afirmación en el caso de los criollos de base española.

\subsection{Lenguas criollas}

Una lengua criolla es aquella que se origina a partir de un pidgin. A su vez, un pidgin es una lengua reducida que se convierte en el principal medio de comunicación entre dos o más pueblos con diferentes lenguas maternas (Holm 1988: 4-5). Tal como apunta Holm (ibid:6):

A creole has a jargon or pidgin in its ancestry; it is spoken natively by an entire speech community, often one whose ancestors were displaced geographically so that their ties with their original language and sociocultural identity were partly broken. Such social conditions were often the result of slavery.

Cuando un pidgin pasa a ser la lengua materna de una generación, se convierte en una lengua criolla. El comercio, pero más frecuentemente la invasión, el desplazamiento forzado y la dominación sociopolítica, son las circunstancias que más impulsan el desarrollo de pidgins y lenguas criollas.

\subsection{Sistema de TMA: universalismo vs. funcionalismo}

Según Wardhaugh (1992: 60), la pidginización de una lengua implica tres procesos generales: a) la simplificación de su morfología y su sintaxis, b) variación fonológica, y c) reducción de funciones. Los procesos de criollización a partir de un pidgin toman la dirección contraria: a) expansión de la morfología y la sintaxis, b) regularización fonológica y c) aumento de funciones.

Al referirse a la evolución de las generalizaciones sobre los sistemas de TMA, Singler (1990) cita los trabajos de Thomson (1961), Taylor (1971) y Muysken (1981). El último resume dos propiedades compartidas por los criollos en relación con las partículas preverbales de TMA de la siguiente manera:

a. Cada criollo tiende a tener partículas que denotan TIEMPO PASADO, MODO POTENCIAL y ASPECTO DURATIVO.

b. Cuando más de una de estas partículas están presentes en el sistema, el orden es siempre TIEMPO-MODO-ASPECTO-VERBO, y las posibles combinaciones de estas partículas conllevan significados fijos y complejos. 
Mediante la comparación de sranán, guyanés, inglés criollo hawaiano y francés criollo haitiano, Bickerton $(1974,1984)$ observa que estos criollos presentan un tiempo ANTERIOR (en lugar de un tiempo PASADO) el cual glosa 'pasado anterior al pasado' en el caso de verbos de acción y 'pasado simple' en el caso de verbos estativos, y agrega a las generalizaciones a. y b. (supra) acerca de las propiedades compartidas, que una forma cero marca el pasado simple en el caso de verbos de acción y el no pasado en el caso de verbos estativos.

Bickerton utiliza el argumento de las propiedades compartidas como explicación a la génesis de las lenguas criollas basada en universales lingüísticos. Sobre la misma base, propone su "hipótesis del bioprograma lingüístico" (language bioprogram hypothesis, LBH), el cual claramente se fundamenta en el "dispositivo de adquisición del lenguaje" (language acquisition device, LAD) propuesto por Chomsky. De acuerdo con la LBH, los criollos emergen en una situación de caos lingüístico en la que los niños tienen un acceso insuficiente a una lengua completa; por lo tanto, a partir de una lengua simplificada que sería el pidgin, desarrollan una lengua completa en virtud de su bioprograma lingüístico, y este fenómeno daría cuenta de las similitudes entre las gramáticas de los distintos criollos. Con el fin de apoyar su LBH, Bickerton descarta evidencia de los criollos fuertes, también llamados endógenos, debido a que en esta situación los niños no están completamente privados de una lengua completa, y en consecuencia, el requisito para que el bioprograma opere no se da. Bickerton incluye datos solamente de criollos de plantación o exógenos, es decir, criollos desarrollados por poblaciones que han sido desplazadas, que hablan diversos vernáculos, y usan el pidgin como principal medio de comunicación.

Singler apunta las siguientes objeciones a la LBH:

a. Como principal medio para explicar las propiedades compartidas de los criollos, un punto débil es la exclusión de datos provenientes de criollos endógenos, ya que el corpus de esa manera resulta sesgado.

b. El sistema de TMA de Bickerton se basa solamente en los criollos de superestrato indoeuropeo. Sería necesario considerar criollos con diferentes superestratos con el fin de verificar si las propiedades compartidas propuestas en efecto se dan en toda clase de criollos.

c. Aún en relación con las cuatro lenguas que Bickerton considera, el argumento de las propiedades compartidas no siempre se sostiene, por ejemplo, el francés criollo haitiano no representa en algunos aspectos el prototipo de su sistema de TMA.

En oposición a la perspectiva de Bickerton, Singler se manifiesta en favor de una teoría del origen de los criollos basada en tres factores:

a. Reconocimiento del papel del substrato, el cual es interactivo con la lengua lexificadora (el superestrato) y con los universales lingüísticos.

b. Factor funcional/pragmático: Si las circunstancias comunicativas en las cuales se desarrolla un criollo favorecen claramente ciertas estrategias lingüísticas, el reconocer este 
hecho contribuiría a comprender mejor tanto la génesis del criollo como las propiedades compartidas por los diversos criollos.

c. Factor tipológico: Una teoría de la génesis del criollo debe tomar en cuenta una amplia gama de pidgins y criollos para explicar no solo las propiedades compartidas, sino también la diversidad entre ellos. La diversidad puede explicarse por: a) las diferencias en cuanto a la situación social en el origen; b) las diferencias en el grado de homogeneidad en los datos de entrada provenientes del substrato, y c) diferencias debidas a sus respectivos substratos y superestratos.

Sin embargo, Singler reconoce que el sistema de TMA propuesto por Bickerton constituye una propuesta fundamental que ha influido en prácticamente toda la investigación posterior relacionada con el sistema de TMA de las lenguas criollas. Una contribución importante es el establecimiento del rasgo de ANTERIORIDAD en la categoría de tiempo. Hay lenguas criollas que confirman el prototipo de Bickerton, con una partícula preverbal de tiempo anterior (por ejemplo el francés criollo haitiano), otros que poseen el tiempo anterior pero en forma de sufijo en lugar de partícula preverbal (criollo de Cabo Verde), y otros que no tienen del todo marcación de tiempo anterior (inglés pidgin kru de Liberia).

El modo irrealis propuesto por Bickerton se refiere a situaciones no reales, es decir, futuro, condicional, subjuntivo, etc. La oposición realis-irrealis también ha sido denominada modo actual-potencial. El aspecto no-puntual de su sistema es denominado más comúnmente aspecto imperfectivo.

En el modelo de Bickerton hay una compartamentalización de tiempo, modo y aspecto, como si se tratara de entidades discretas. En la opinión de Singler, hay fuertes correlaciones entre pares de categorías, en particular entre tiempo futuro y modo potencial o irrealis, entre tiempo no futuro y modo actual o realis, entre tiempo presente y aspecto imperfectivo, y entre tiempo pasado y aspecto perfectivo. La interacción entre tiempo y aspecto, principalmente, pero también entre aspecto y modo, da origen a sistemas de TMA mucho más complejos que el prototipo propuesto por Bickerton.

Singler (ibid: xiv) concluye que :

(...) while pidgins and creoles share critical properties, they also show a far reaching diversity. Thus to relate creole TMA to creole genesis and TMA theory it is necessary to consider not only unity but also diversity in TMA in pidgins and creoles.

Una visión intermedia es la de Givón (1984: 291), quien sigue a Bickerton al argumentar que cuando los niños adquieren una lengua pidgin de sus padres,

(...) they extract the only reasonable stable feature from the Pidgin, the lexicon, and then go on to invent the Creole grammar from scratch, presumably relying in their human-universal faculty of language/communication. 
Sin embargo, Givón orienta su discusión acerca del sistema de TMA basándose en la idea de que el uso de las partículas de TMA está pragmáticamente determinado, con lo cual su posición coincide con la de Singler.

\subsection{Prototipo del sistema de TMA de Bickerton}

TMA:

Los siguientes son los rasgos propuestos por Bickerton (1974: 5-6) del sistema de

a. La forma cero marca el pasado simple en verbos de acción y el no pasado en verbos estativos.

b. Un marcador de anterioridad indica pasado-anterior-al-pasado en verbos de acción y pasado simple en verbos estativos.

c. Un marcador de aspecto irrealis indica "situación no-real", es decir, futuro, condicional, subjuntivo, etc., en todos los verbos.

d. Un marcador de aspecto no puntual indica aspecto durativo o iterativo en verbos de acción, y es indiferente en cuanto a la distinción pasado-no pasado; este marcador no se da junto a verbos estativos.

e. Todos los marcadores se dan en posición preverbal.

f. Todos los marcadores se pueden combinar, pero guardan un orden invariable: anteriorirrealis-no puntual.

g. El significado de anterior + irrealis es "condición no realizada del pasado".

h. El significado de anterior + irrealis + no puntual es una condición no realizada en el pasado y de naturaleza no puntual, algo semejante a "si tan solo hubiera hecho Y".

El significado de anterior + no puntual es "acción no puntual ocurrida en tiempo no real", por ejemplo un futuro progresivo: estaré haciendo $Y$.

\section{Criollos de base española}

Hancock (1971) describe brevemente 12 criollos y pidgins de base española (ver también Crystal 1987: 338-9):

En América del Norte: pachuco o pochismo, lengua de contacto español-inglés en Arizona y California.

En América Latina: español-nahuatl criollizado (Nicaragua, siglo XVI, extinto); español pidgin utilizado por algunas tribus indígenas de Venezuela; español criollizado de San Basilio de Palenque, Colombia; cocoliche (también llamado lunfardo y rioplatense) es un español italianizado que se usa en Buenos Aires y levemente pidginizado ${ }^{1}$. frañol, una lengua de contacto francés-español en Buenos Aires, y papiamento, lengua que Hancock define como un criollo español derivado de un pidgin portugués previo (ibid: 513), que se habla en Curaçao, Bonaire y Aruba.

En Europa: inglés de escalerilla, pidgin de base inglesa y española utilizado en los puertos españoles del Mediterráneo. 
En Filipinas: chabacano o zamboagueño, criollo de base española con influencia de tagalo y cebuano y que se habla en Zamboanga; davaueño o "español abakay" que se habla en Davao; ternateño, criollo utilizado entre soldados españoles y la comunidad portuguesa de Moluccas, Ternate, y finalmente ermitaño, hablado en el área de Manila.

En cuanto a otras regiones en donde se habla el español, no se ha encontrado ningún desarrollo propiamente criollizado de esta lengua. Un ejemplo de español no criollizado se habla en Guinea Ecuatorial:

The only Spanish speaking area in Sub-Saharan Africa is Equatorial Guinea; Guinea's Spanish however is not creolized but stands in a stable situation of bilinguism between Spanish and Western African languages. (Lipski 1984: 69)

Otro ejemplo es el español que se habla en el Valle del Chota en las tierras altas de Ecuador, donde Lipski (1987: 159) no pudo comprobar la existencia, al menos en la actualidad, de un español criollizado:

This finding does not preclude the prior existence of partially Africanized Spanish in the Chota region (...), but it does set back the dates for the gradual decreolization that would have given rise to the present popular, but noncreolized, Choteño Spanish.

Un último ejemplo, interesante para el estudio de los criollos de base española pero que tampoco representa una verdadera variedad criolla, es el habla de los llamados negros congos de Panamá. Este dialecto del español investigado por Lipski (1989) se utiliza solo en ceremonias tradicionales y solamente por parte de ciertos miembros de la comunidad. Se basa en el español popular y "ha sido modificado y deformado al punto de inhibir su comprensión por parte de los no iniciados" (ibid: 1). Acerca de su situación particular de uso, Lipski (ibid: 6-7) señala:

The congo ceremonies and games are characterized by the consumption of extraordinary quantities of alcohol (...) Since the congo dialect is not frequently used outside of Carnival season, it has naturally come to be identified with festive atmosphere which surrounds Carnival season, to the extend that many congo practitioners, especially the recognized masters of congo dialect, claim that they cannot speak in this mode unless they are bien sudao "loaded".

En relación con el origen de los estudios de criollos de base española, de Granda (1968a) señala que, hasta la fecha de su propio estudio, solamente el papiamento y los criollos filipinos habían sido incluidos en los estudios de lingüística hispánica. Pero como resultado del desarrollo de la teoría monogenética y de la criollística en general, se estableció la existencia de otras hablas criollizadas del español. En particular, de Granda menciona el artículo de José Joa- 
quín Montes titulado "Sobre el habla de San Basilio de Palenque (Bolívar, Colombia)" (Thesaurus, XVII, 1962, 446-50) y el trabajo de M. Álvarez Nazario "Criollo puertorriqueño vivo en el siglo XIX: El elemento afronegroide en el español de Puerto Rico" (San Juan, 1961).

De Granda (1968b) enlista los siguientes rasgos morfosintácticos compartidos por los criollos de base española considerados en este trabajo:

a) Invariabilidad de sustantivos y adjetivos con respecto a las categorías gramaticales de género y número.

b) Identificación de los pronombres personales y posesivos (con unas pocas excepciones en papiamento y los criollos filipinos).

c) Ausencia de elementos sintácticos conjuntivos.

d) Uso del pronombre vos de segunda persona ${ }^{2}$.

e) Construcción de $t a+$ infinitivo para el tiempo presente.

f) Uso del esp. tener como estativo existencial.

g) Existencia del pronombre de tercera persona ele.

h) Invariabilidad del artículo indefinido un.

En sus conclusiones, de Granda afirma que estas correspondencias morfosintácticas entre palenquero y el habla bozal de Puerto Rico por una parte, y entre papiamento y los criollos filipinos por otra, muestran que todas estas variedades lingüísticas están tipológicamente relacionadas y representan un grupo dentro de las lenguas criollas. (De Granda señala la existencia de estos rasgos también en algunos criollos de base portuguesa, con el fin de defender la teoría monogenética, la cual postula un proto-criollo portugués como el origen de todos los criollos; sin embargo, este aspecto no es relevante para el propósito del presente trabajo.) Como es evidente, el único rasgo de la lista de de Granda que concierne al sistema de TMA es (e), la construcción $t a+$ infinitivo para el tiempo presente.

Lipski (1985) resume un conjunto más general de propiedades compartidas por los criollos de base española:

a) Severa reducción de la morfología verbal: los verbos son reducidos a la forma infinitiva con tendencia a la pérdida de la -r final.

b) Reducción de la morfología nominal. Este rasgo se refiere al rasgo (a) de la lista de de Granda.

c) Modificación y reducción del sistema pronominal. Este rasgo se refiere al rasgo (b) de de Granda. Lipski añade la fusión de las formas pronominales en los casos de sujeto y objeto directo.

d) Reducción del uso de proposiciones.

e) Reducción de la complejidad sintáctica. Este rasgo se refiere a la evitación de cláusulas subordinadas, pronombres relativos, etc.

f) Reducción e inseguridad fonológicas. Este rasgo incluye una serie de procesos, por ejemplo la pérdida de oposiciones fonológicas, distribución alofónica incierta, etc. 
Habiendo esquematizado estas características generales compartidas por los criollos de base española, podemos ahora dirigirnos al dominio concreto de sus sistemas de TMA con el fin de determinar si las partículas preverbales que codifican oposiciones de TMA se ajustan al prototipo propuesto por Bickerton.

\section{El sistema de TMA en tres criollos de base española}

\subsection{Palenquero (Colombia)}

A pesar de que existen algunas referencias sobre esta lengua (ver de Granda 1978), la información publicada sobre ella es extremadamente escasa. Presento aquí datos recogidos en Bickerton y Escalante (1970) $)^{3}$.

De acuerdo con los autores, el palenquero "comparte con la mayoría de los criollos los morfemas que sirven como marcadores de tiempo o aspecto" (p. 257-8).

3.1.1. Partícula ta

Marca aspecto continuativo (CONT) pero parece limitado al tiempo presente (PRES):

1) I ta semblá patija aora.

yo CONT/PRES sembrar sandía ahora

'Yo estoy sembrando sandía ahora.'

2) I ta ablá bo.

yo CONT/PRES hablar vos

'Yo te estoy hablando.'

3) Anda panda gaina, que ta cumé to aló.

anda espanta gallina, que CONT/PRES comer todo arroz

'Anda espanta la gallina, que se está comiendo todo el arroz.'

3.1.2. Partícula tan (de tando 'ir')

Marca tiempo futuro (FUT):

4) I tan semblá aló.

yo FUT sembrar arroz

'Yo voy a sembrar arroz.'

\subsubsection{Partícula ase}

Relacionada con esp. hacer, marca el aspecto habitual (HAB):

5) Toos los años hace parí un moná. todos los años HAB parir un hijo

'Todos los años doy a luz un hijo.' 
6) Bo ase kumé kane?

vos HAB comer carne

¿Vos acostumbrás comer carne?’

\subsubsection{Partícula a}

Marca aspecto completivo (COMPL), pero algunas veces está ausente en el caso de acciones completas:

7) Ele a pelé.

ella COMPL perder

'Ella se perdió.'

Pero:

8) I miná bo nu

yo mirar vos NEG

'No te vi.'

Los autores sugieren que la ausencia de $a$ en oraciones negativas puede ser resultado de una transformación que impulsa la supresión de marcadores de tiempo-aspecto, en casos en que el marcador de negación nu está presente.

La partícula a aparece con verbos estativos como ten 'tener', ten ke 'tener que', sabé 'saber', kelé 'querer' en la expresión de situaciones presentes:

9) Bo a sabé ané pule ablá ku bo nu.

vos PART saber ellos poder hablar con vos NEG

'Vos sabés que ellos no pueden hablar con vos.'

10) Ele a ten kala bunito.

ella PART tener cara bonito

'Ella tiene una cara bonita.'

11) I a ten uto chiquitito.

yo PART tener otro chiquitito

'Yo tengo otro chiquito.'

12) Bo a ten ke buká uto xende.

Vos PART tener que buscar otro gente

'Vos vas a tener que buscar a otra persona.' 
En (12), los autores consideran que $a$ podría ser una forma reducida de ta. A mí me parece que podría ser más bien una síntesis de la construcción de futuro "ir a + infinitivo" del español. Cp. pal. bo a ten que..., esp. vos vas a tener que ...; sin embargo, más datos serían necesarios para aclarar el punto.

\subsubsection{Marcación cero}

Algunos ejemplos extraídos de Bickerton y Escalante demuestran que el tiempo presente frecuentemente tiene marcación cero:

13) E la rie.

es la diez

'Son las diez.'

14) Bo miná ke bo e ri aki nu

vos mirar que vos es de aquí NEG

'Vos ves que vos no sos de aquí.'

En oraciones negativas:

15) I kelé miná bo nu yo querer mirar vos NEG

'No quiero verte.'

16) Mailo mi quelé nu

marido mí querer NEG

Mi marido no quiere que yo...'

El pasado también puede presentar marcación cero en el caso de acciones, como puede verse en el siguiente ejemplo (al menos tal como lo traducen Bickerton y Escalante):

17) ke bo tre mi

qué vos traer mí

¿Qué me trajiste?’

\subsection{6. ¿Generalizaciones?}

Los datos disponibles son demasiado escasos como para hacer generalizaciones. Sin embargo, podría investigarse la hipótesis de si en esta lengua la marcación cero se ajusta al prototipo de Bickerton, es decir, si marca pasado simple en verbos de acción (ejemplo 17) y no pasado en los verbos estativos o al menos no típicamente de acción (ejemplos 13-16). A mí me parece que $a$ tiene la tendencia de oponerse a la marcación cero, en el sentido de que podría marcar acciones pasadas afirmativas puntuales (ejemplo 7) y no-acciones no pasadas afirmativas durativas (ejemplos 9-11) (un estado puede ser no durativo, como por ejemplo en el caso de 
estado pasado perfectivo fue del español, el cual es puntual). Sin embargo, no podría defenderse esta proposición sobre la base de un corpus tan reducido.

Por otra parte, parece claro que ta marca aspecto durativo (este podría ser el marcador de aspecto no-puntual de Bickerton, el cual no co-aparece con verbos estativos), y que ase marca aspecto habitual, si puede afirmarse esto con base en dos únicos ejemplos.

Con respecto a otros rasgos del prototipo de Bickerton, la única característica que se manifiesta claramente sobre la base de estos datos es que las partículas son preverbales. No se puede analizar combinaciones de partículas ya que no se presenta ningún caso en este corpus, así como tampoco se puede establecer ningún hecho con respecto a las partículas de anterior ni de irrealis.

\subsection{Papiamento (Curaçao, Aruba y Bonaire)}

\subsubsection{La descripción de Andersen del sistema de TMA del papiamento}

Andersen (1990: 60) señala que "el papiamento ha sido frecuentemente mencionado como contraevidencia para la validez del bioprograma propuesto por Bickerton”. Andersen (loc. cit.) resume así la evidencia para esta afirmación:

Papiamentu apparently stands out as an exception (to Bicketon's prototype) in that it has no anterior marker, the irrealis marker $(l o)$ does not keep its predicted position, and the aspectual system is complicated by the fact that it is clearly encoded only in past reference and appears to mirror the Spanish perfective:imperfective opposition. In other words, the Papiamentu tense-modality-aspect markers do not fit Bickerton's paradigm.

El conjunto de partículas preverbales de TMA del papiamento es el siguiente (Andersen, 1990: 67):

TIEMPO-ASPECTO (T-A)

$\begin{array}{ll}\text { ta: } & \text { imperfectivo: } \\ & \begin{array}{l}\text { Wan ta kome bonchi. } \\ \text { 'Juan come/está comiendo frijoles.' }\end{array} \\ a: \quad \text { Wan a kome bonchi. } \\ \end{array}$

tabata: pasado Wan tabata kome bonchi.

imperfectivo: 'Juan estaba comiendo/solía comer frijoles.'

sa: habitual:

Wan sa kome bonchi.

'Juan suele comer frijoles.' 


\section{MODO (M)}

lo: irrealis:

Wan lo kome bonchi.

'Juan comerá frijoles.'

0: $\quad$ subjuntivo: $\quad$ (Si) Wan kome bonchi

(Si) Juan come frijoles...'

Andersen señala que los enfoques tradicionales consideran ta como un marcador de tiempo presente, a como 'pasado perfectivo' y tabata como 'pasado imperfectivo', y admite que tal descripción corresponde relativamente bien a la variedad educada formal, pero, tal como argumenta (ibid: 68),

(...) in basilectal Papiamentu (...) there is an opposition between tense-neutral $t a$ (imperfective) and $a$ (perfective). Although $t a$ is certainly the typical "present" marker (...) this is a consequence of its status as an imperfective marker. And perfective $a$ usually has past time reference, but not because $a$ is intrinsecally a "past" marker.

Con respecto a tabata, añade (loc. cit.):

Imperfective tabata functions as a past-marked variant of $t a$ and is used in spontaneous discourse only to anchor an event clearly in the past when otherwise the use of $t a$, which is tense-neutral, would be misleading.

Diversos papiamentistas escriben al parecer erróneamente la partícula tabata, dando la impresión de que se pronuncia [tabáta]. En cuanto a su interpretación en relación con $t a$, Yang (1988: 35) argumenta:

Birminham [1970] denomina la partícula ta 'función de presente', pero es un hecho que su aparición no está limitada al presente. Birminham mismo reconoce que tába ta (que él escribe / 'tabata) se puede analizar en tába más ta (1970: 87), pero trata esta combinación como una unidad que llama 'función de imperfecto'. Si se considera que tába puede presentarse sin $t a$ con el valor de pretérito en secuencia con temas verbales de una pequeña clase indiferente a la oposición aspectual perfectivo/imperfectivo su tratamiento es claramente insostenible.

\subsubsection{Partícula ta:}

Tiene referencia al pasado, tanto en tiempo genérico como con significado progresivo. "Lo que $t a$ codifica no es 'presente', 'presente simple' ni 'presente progresivo', sino más bien un aspecto imperfectivo neutral en cuanto a tiempo" (ibid: 69). Los ejemplos que Andersen da son: 
18) E ta bai ku bus.

él T-A va con bus

'El viaja en bus.'

19) Nan ta yora pasobra nan ta pober.

ellos T-A llora porque ellos COP pobre

'Ellos lloran porque son pobres.'

\subsubsection{Partícula a}

Se refiere a "una acción que se asume como ocurrida en algún momento previo al tiempo de referencia presente, dentro del texto" (Andersen loc. cit.):

20) Bosonan no a siña na skol?

vos-pl NEG T-A enseñar en escuela

¿Ustedes no han aprendido (esto) en la escuela?’

\subsubsection{Partícula tabata}

Como $t a$, implica acción durativa, sea esta iterativa, habitual o progresiva.

21) E hòmber tabata yena awa ku makutu.

el hombre T-A llenar agua con balde

'El hombre estaba llenando de agua un balde.'

\subsubsection{Partícula sa}

Como ta, es un marcador de imperfectivo, en particular con significado habitual:

22) E' n ta hende ku sa keda bashí.

él NEG COP gente que T-A quedar vacío

'Él no es persona que se queda con las manos vacías.'

Con respecto a sa, Andersen (ibid: 71) agrega:

$\mathrm{Sa}$ has a different status from the other tense-aspect markers. First of all, unlike $t a, a$, and tabata, sa is not obligatory. Furthermore, $s a$ is also an independent verb, as in Mi sa hulandes 'I know Dutch' and, with an auxiliary function, $M i$ sa landa 'I know how to swim'. The two meanings "know" and "be accustomed to" are clearly distinct; and I have chosen to include $s a$ among the tense-aspect markers in spite of its more marginal status.

Se debe observar, con respecto a esta caracterización de $s a$, que aparentemente Andersen no logra reconocer que $s a$, en la función que él denomina 'auxiliar', es simplemente el ver- 
bo saber, el cual tiene exactamente los mismos usos que en papiamento: esp. yo sé holandés, yo sé nadar. Por otra parte, sa, en el sentido de 'estar acostumbrado a', al menos en el único ejemplo presentado por Andersen, me parece una forma no relacionada en forma alguna con $s a$ 'saber'; más bien parece ser la partícula impersonal se. En la traducción de (22) él no es persona que se queda con las manos vacías, el sentido de se queda es también habitual, pero no por la presencia de $s e$, sino porque es parte de una cláusula relativa restrictiva (nótese que en el ejemplo en papiamento sa keda también pertenece a una cláusula relativa). En cualquier caso, no afirmo que no exista en papiamento un marcador de aspecto $s a$, sino que sugiero la necesidad de presentar más ejemplos como evidencia.

\subsubsection{La caracterización de Ferrol del sistema de TMA del papiamento}

Ferrol (1982) reconoce tres partículas preverbales en el sistema verbal del papiamento, las cuales conllevan tres categorías aspectuales binarias:

-continuo vs. no continuo

-perfectivo vs. imperfectivo

-real vs. hipotético (no real)

\subsubsection{Partícula ta}

Marca aspecto continuativo, mostrando que la acción verbal es progresiva. Taba-ta se considera aquí como una subcategoría que marca tiempo pasado:

23) mi ta kome 'yo como, estoy comiendo'

24) mi tabata kome 'yo estaba comiendo, solía comer'

\subsubsection{Partícula a}

Marca aspecto perfectivo, es decir acción completa ya sea en relación con el momento presente o en relación con cualquier otro tiempo.

25) mi a kome 'yo comí, he comido'

\subsubsection{Partícula lo}

Expresa el tiempo hipotético de una acción cuya realidad es desconocida. Como tal, incluye la referencia al futuro:

27) lo mi ta kome

29) lo mi tabata kome 'yo habría estado comiendo' 
Estos ejemplos proveen evidencia a la afirmación de Andersen de que el marcador de irrealis lo no aparece en la posición predicha por el prototipo de Bickerton: dicha partícula, a pesar de ser preverbal aparece delante del sujeto, y esta situación diverge notablemente de la estructura normal del sistema de TMA criollo. Nótese, sin embargo, que en los ejemplos tomados de Andersen que aparecen en 3.2.1. supra, la partícula lo aparece en la posición preverbal predicha por el prototipo 4 .

A pesar de que Ferrol no se refiere particularmente a este punto, afirma que estos ejemplos “demuestran claramente que la 'conjugación' de los verbos en papiamento no corresponde ni al español ni al portugués. Los moldes que se siguen son muy probablemente africanos" (ibid: 54). Me referiré a este punto en las conclusiones de este trabajo.

\title{
3.2.3. Generalizaciones
}

En relación con el conjunto de características propuestas por Bickerton, se puede ver que el papiamento es divergente en varios aspectos. Pero este hecho ha sido ampliamente discutido por el mismo Bickerton. En este sentido, vale la pena consignar aquí la conclusión de Andersen (1990: 89):

\begin{abstract}
Bickerton (1981: 85-88, 1984: 177) has tried hard to account for the ways in which Papiamentu deviates from the prototypical TMA system. His only source for Papiamentu, however, is Goilo (1972), which is a good textbook, but was not meant to be a sophisticated linguistic treatise. In fact, Goilo is inadequate as a source on basilectal Papiamentu (and Bickerton's bioprogram only concerns the basilect); as one would expect in such a textbook, it more accurately reflects the formal usage of well-eduacted bilingual and multilingual speakers of Papiamentu. It is ironic that Bickerton, of all people, should overlook this fact, given his pioneering work on the creole continuum (Bickerton 1975, among other works). This article has shown that, when attention is primarily focused on basilectal Papiamentu, the Papiamentu tense-aspect system is much closer to Bickerton's bioprogram than he realizes. The primary opposition, (...) is that of a tense-neutral imperfective $(t a)$ vs. a tense-neutral perfective $(a)$, which is what the bioprogram predicts, at least in terms of the semantics of the system, although not with respect to the expected zero marking of perfective aspect.
\end{abstract}

Podemos examinar un poco más otros de los rasgos del prototipo de Bickerton. Una de las divergencias más importantes, como hemos visto, es la ausencia de un marcador de anterioridad. El marcador de irrealis está presente, aunque en una posición no esperada; el aspecto no-puntual también está marcado mediante una partícula preverbal, y todos los marcadores ocupan una posición anterior al verbo. La combinación de Bickerton de irrealis más no-puntual aparece en este orden, pero otras combinaciones de marcadores no aparecen en papiamento, debido a la ausencia de un marcador de anterioridad. Sin embargo, Ferrol pro- 
vee un ejemplo de co-aparición del irrealis lo más el no-puntual o durativo ta de acuerdo con el cual el significado, tal como predice el modelo de Bickerton, es un futuro progresivo: lo mi ta kome 'estaré comiendo'.

\subsection{Criollos filipinos de base española}

Whinnom (1956) describe el sistema verbal de tres criollos de base española desarrollados en distintas áreas de las Filipinas. Utiliza el término "vernáculos de contacto" (contact-vernacular) para referirse en general a estos criollos: ternateño (el primero en desarrollarse) y las variedades derivadas caviteño y ermitaño; el zamboagueño, relativamente independiente del anterior, y su variedad derivada davaueño. Whinnom afirma que "es claro que estos cuatro vernáculos son esencialmente uno mismo, todos descendientes más o menos directamente del ternateño" (ibid: 91), y uno de sus principales argumentos para apoyar esta afirmación es que todos comparten exactamente el mismo sistema de TMA:

In general the contact-vernacular verb consists of a 'word-base' derived from the Spanish infinitive which is modified by three particles to form the three modes. (Whinnom: loc. cit.)

Estas partículas son ta, ya y de. Whinnom observa que a pesar de que estas pueden ser traducidas como presente, pasado o futuro en las lenguas indoeuropeas, esto "solamente revelaría una aproximación a la realidad" (ibid: 92).

\subsubsection{Partícula ta}

Indica tiempo presente pero solo con aspecto durativo (DUR). Ejemplos:

30) Ta sumí el sol. ${ }^{5}$

DUR sumir el sol

'El sol se está poniendo.'

31) Nisós ta comé.

nosotros DUR comer

'Nosotros estamos comiendo.'

32) Yo ta iscribí cuando ele ya vení.

yo DUR escribir cuando ella ya venir

'Yo estaba escribiendo cuando ella llegó.'

33) ...como ta llorá el mana estrella.

como DUR llorar el PL ${ }^{6}$ estrella

'...mientras lloran las estrellas.' 
34) El mana dolor ta pasá.

el PL dolor DUR pasar

'Las penas pasan.'

\subsubsection{Partícula ya}

Es el marcador de puntualidad (PUNT), es decir, conlleva aspecto perfectivo indicando una acción completa. Ejemplos:

35) Yo ya comprá plátanos ayer.

yo PUNT comprar plátanos ayer

'Yo compré plátanos ayer.'

36) Nisós ya andá na Manila. nosotros PUNT andar en Manila

'Nosotros fuimos a Manila.'

37) Ya cugí un piscao grande-grande.

PUNT coger un pescado grande-grande

'Cogí un pescado muy grande.'

38) No ya podé escondé el dolor.

NEG PUNT poder esconder el dolor

'No pudo esconder el dolor.'

En relación con (38), Whinnom (ibid: 34) explica:

The position of the negative, which in Spanish preceeds the verb, shows how intimately the particle $y a$ has come to be regarded as part of the verb.

\subsubsection{Partícula de (o ay)}

Es el marcador de modo contingente (CONTIN) y se utiliza para la referencia al tiempo futuro, pero también para expresar intención en casos en que el español usaría el subjuntivo: "The de of Ermitaño indicates no more than an intention, a futurity which may be either present or past" (Whinnom ibid: 39). Ejemplos:

39) dónde tú de andá? dónde tú CONTIN andar ¿A dónde irás?' 
40)
cómo de llorá yo?
cómo CONTIN llorar yo
'Por qué he de 7 llorar?'

41) ele ta gualdá para cuando Pelisa de casá

él DUR guardar para cuando Pelisa CONTIN casar

'El ha/había ahorrado para cuando Pelisa se case/casara.'

\subsubsection{Recursos para enfatizar}

Este sencillo sistema puede expresar significados más complejos mediante la utilización de los siguientes tres recursos, según señala Whinnom (ibid: 93):

a. la reduplicación del verbo se usa para intensificar el aspecto progresivo: nisós ta comé-comé 'Nosotros estamos comiendo y comiendo.'

b. la forma adverbial y posverbal ya se usa para enfatizar el aspecto puntual: ele de cabá ya su trabajo cuando vos de vení 'él habrá terminado su trabajo para cuando vos llegués' (cf. ele de cabá su trabajo 'él terminará su trabajo.');

c. uso del verbo cabá: tiene la misma función que el adverbial ya, pero agrega aún más fuerza. Whinnom no provee ejemplos de este uso especial del verbo; en el ejemplo (b) supra cabá es el verbo principal de la oración.

\subsubsection{Generalizaciones}

Whinnom (ibid: 94-5) resume el sistema de TMA de los criollos filipinos del siguiente modo:

The form of the contact-vernacular verb is virtually that of the Spanish infinitive, (...) and the auxiliary particles $t a, y a$, and $d e$ are Spanish (or Portuguese) in origin. But the psychology of the verb is essentially Malay and not Indo-European, Tagalog, not Spanish.

The verb has two modes, contingent and actual, the latter of which is further divided into two aspects, durative and punctual. With the assistance of the Spanish adverbial ya a contingent punctual may also be formed; while the use of the Spanish locution acabar de in the form of cabá de is also used to express perfective notions.

Queda claro que el pasado simple en los verbos de acción no se marca mediante cero sino mediante ya. En cuanto a los estativos, el verbo ser no aparece: tú bata 'tú (eres un) niño'; ele tan guapa 'ella (es) muy guapa'; el estativo español estar fue reducido y contraído con los deícticos espaciales allí, allá: el manzana tallí na mesa 'la manzana está allí en la mesa'. En otros estativos el no-pasado se marca con ta: na pondo del nubes que ta sostené el mar 'en el fondo de las nubes que el mar sostiene'.

El sistema carece de un marcador de anterioridad, pero sí presenta el marcador de aspecto irrealis, así como también el marcador no-puntual o durativo ta. Este, sin embargo, puede 
co-aparecer con verbos estativos: no ta tené ya amor na mundo 'yo ya no tengo amor en este mundo', ejemplo en el cual ya es adverbial.

Todos los marcadores aparecen en posición preverbal. Whinnom no se refiere a ningún tipo de combinación. En los textos aparece un ejemplo en el que los marcadores de irrealis y puntual co-aparecen con el verbo: (Contexto: Dónde tú de andá? ‘¿Adónde vas?’) De salí ya yo con ustedes. Desgraciadamente Whinnom no provee la traducción de los textos que publicó. En cuanto a esta construcción, señala, sin embargo: "This is an interesting form, indicating as it does future and perfective action at the same time" (p. 41). De acuerdo con esto, la posible traducción en este caso sería: 'Yo me habré ido con ustedes'8.

Otra combinación encontrada es ya + ta. En caviteño: tres hora ya ta jilví-jilví 'ha estado hirviendo durante tres horas'. Este ejemplo muestra que la partícula ya conlleva también el significado del marcador de anterioridad de Bickerton, en particular si consideramos el significado de su combinación anterior + no-puntual: una acción durativa o serie de acciones no-durativas que ocurren ya sea antes de algún otro evento en discusión, o durante un periodo de tiempo considerado como definitivamente cerrado.

Otro ejemplo de esta combinación es (caviteño):

42) (Ta cuidá di manga-puelco chiquitito,)

DUR cuidar de cerdo chiquitito

'((Ella) cuida de los cerdos pequeños,)

y cuando grande,

y cuando grande

y cuando (están) grandes,

ya ta vindí ele para tiní qui gastá.

PUNT DUR vender ella para tener que gastar

los vendería para tener (dinero) qué gastar.'

No fue posible encontrar ninguna combinación de irrealis + no-puntual $(d e+t a)$ en los textos de Whinnom.

\section{Conclusiones}

Los sistemas de TMA de los tres criollos considerados pueden ahora compararse en términos de las partículas preverbales que presenta cada uno de ellos: 


\begin{tabular}{|c|c|c|}
\hline CRIOLLOS: & Palenquero & Papiamento \\
\hline
\end{tabular}

filipinos

\section{TIEMPO-ASPECTO}

durativo

habitual

puntual

MODO

irrealis ta

(+presente)

ase

$a$

tan

(+futuro) ta

tabata (+pasado)

$s a$

$a$

ya

lo

de ta

.


I: $\quad$ Y a ten uto chiquitito un catambiquito po ai que hace ñamá Joselís.

'Yo tengo otro chiquito, un "catambuquito" por ahí que se llama José Luis.'

E: $\quad$ esp: Todos los años uno.

I: $\quad$ Toos los años hace parí un moná. E que y atá güeno; vac'atá güeno; no pierdo tiempo.

Todos los años tengo ${ }^{9}$ un "moná". Es que estoy buena, la vaca está buena, no pierdo tiempo.'

E: $\quad$ esp: Tienes que tomar remedio.

I: No - mailo mi quelé nu. El no me quelé mi asina. Güeno que y parí un moná to los a... anda panda gaina, que ta cumé to aló!

'No, mi marido no lo quiere. El no me querría a mí así. Bueno que (yo) tenga un “moná” todos los a... andá espantá la gallina, que está comiéndose todo el arroz!'

El texto muestra que la informante mezcla español y palenquero. Tal como lo señalan Bickerton y Escalante (loc. cit.):

The alternation between, or actual mixing of, Spanish and Palenquero forms is very apparent. For instance, terminal $n u$ in one sentence is followed by preverbal no in the next; in the latter sentence, the object pronoun is rendered twice, by preverbal Spanish $m e$ and postverbal Palenquero $m i$; a Palenquero sentence -vac' atá güeno, ${ }^{10}$. with unstressed vowel elision and absence of gender concord- is followed immediately by an almost purely Spanish one no pierdo tiempo.

Papiamento: Un fragmento de un cuento tradicional (Andersen 1990: 82-3).

El a haña un chuchubi meimei di dos palu grandi.

en medio de dos palos grandes.

El ha encontrado un "chuchubi" l1

Ora e chuchubi $0^{12}$ lanta,

"Cuando el "chuchubi" se levante,

for di e palu di-pa riba

'se vaya del palo de arriba,

ku e 0 baha na esun di pa bou,

'si se baja hacia ese (palo) de abajo

e palo di pa riba ta muri,

'el palo de arriba se muere,

esun di pabou ta biba.

'y ese de abajo vive.

Ora e 0 lanta for di esun di pa bou,

'Cuando él se levante, se vaya del (palo) de abajo

ku e 0 baha na esun di pa riba,

'si se posa en ese de arriba,

esun di pa bou ta muri,

'ese de abajo muere,

esun di pa riba ta biba.

y ese de arriba vive.' 
Este texto indica que mientras el papiamento refleja léxicamente su superestrato, el comportamiento de la gramática y particularmente el sistema de TMA es completamente diferente, tal como es el caso de todos los demás criollos conocidos. Dijimos anteriormente que este comportamiento del sistema de TMA ha sido atribuido a la influencia de lenguas africanas que constituirían substratos de los criollos:

La lengua Nkonya, de Togo, tiene un aspecto habitual, representado exactamente por la partícula 'ta'... El Awutu y el Akan tienen un prefijo 'le' I'láá' para el futuro intencional. Y para el perfecto, el Nkonya y el Akan tienen un prefijo -a-. Por consiguiente, mientras la interpretación hispano- portuguesa suponía partículas de muy diferente valor tomadas al azar de cualquiera de las dos lenguas y alternando en forma y función, la hipótesis de un origen africano nos muestra un sistema coherente que pasó tal cual era al papiamento. Evidentemente se requerirán todavía muchas indagaciones para situar precisamente el origen de estas partículas, pero desde ya creemos poder afirmar que en papiamento provienen de alguna lengua de la Costa de Guinea, tanto por sus funciones como por sus formas." (Rona 1970: 14, citado en Ferrol 1982: 54).

En cuanto al argumento de que los sistemas de TMA de estos criollos se derivan de la gramática de lenguas africanas, es de rigor hacer la observación obvia de que las gramáticas de las lenguas africanas varían considerablemente, por lo que no es posible establecer un paralelo entre el sistema de TMA criollo y un supuesto sistema de TMA africano. Como ilustración, presento infra datos de la lengua wolof, de la familia niger-congo, hablada en Senegal, en la costa occidental de África, lugar de origen de muchos de los esclavos traídos a América, y los comparo con ejemplos de papiamento tomados de Ferrol (c. 3.2.2): ${ }^{13}$

Pap. mi ta kome 'yo como/estoy comiendo.'

Wol. ma ngi lek

yo PRES comer

'yo como (presente neutro)'

'yo comeré (futuro inmediato)'

Wol. da ma i lek

PROG yo PRES comer

'yo estoy comiendo (presente progresivo)'

'yo como (habitual)'

Pap. mi tabata kome 'yo comí/he comido.' 
Wol. da ma den lek

PROG yo PAS comer

'yo estaba comiendo (pasado progresivo)'

Wol. den na ${ }^{14} e k$

PAS yo comer

'yo solía comer (pasado habitual)'

Pap. mi a kome 'yo comí/he comido'

Wol. da ma leken

PERF yo comer.PERF

'yo comí (pasado perfectivo, expresado mediante

la combinación de la partícula $d a$ y el sufijo -en.)

Pap. lo mi kome 'yo comeré'

Wol. di na lek

FUT yo comer

'yo comeré'

Pap. lo mi a kome 'yo habré comido'

Wol. dinfik ma lek

será yo comer

'será el caso que yo habré comido'

Puede verse que a pesar de que se comparte el rasgo de la posición preverbal de las partículas que expresan TMA, este no es siempre el caso, y la combinación de partículas no muestra ningún paralelismo. Finalmente, debe notarse que hay mucha más semejanza entre los sistemas de TMA de los tres criollos comparados, que entre el sistema del papiamento y el sistema del wolof.

Ermitaño: Un fragmento del texto literario "Na maldito arena", escrito por Jesús Balmori y publicado por primera vez en The Philippine Review de abril de 1917. Whinnom (1956: 23) explica acerca de este texto:

The story Na Maldito Arena is clearly the work of a littérateur, (...) it seemed better to print this piece of sophisticated simplicity, written in comparatively pure Ermitaño, with the warning that it is not typical of contact-vernacular literature, than to transcribe a genuine folk-tale in highly contaminated dialect. 
Esta explicación nos permite considerar el fragmento como un buen ejemplo del vernáculo de contacto filipino, en particular del ermitaño.

Y el viejo ta pensá na su vida, peliz agora,

'Y el viejo piensa en su vida, feliz ahora

masque el madre de Pelisa ya morí de viejo.

'que la madre de Pelisa murió de vieja.

No namás ${ }^{15}$ dos mil pesos el que ta gualdá ele na alcancía.

'No menos de dos mil pesos guarda él en su alcancía

dos mil pesos que ya conveltí en cualta

'dos mil pesos que ya convirtió en cuartos

todo el mana de escamas de plata que ya calgá su rede

'todos los pescados ${ }^{16}$ que cargaron sus redes

na celca de quince años

'en cerca de quince años

y que ele ta gualdá para cuando Pelisa de casá...

'y que él guarda para cuando Pelisa se case...

Pelisa! Sús María!

‘Pelisa! ¡Jesús María!

Ta pijá bueno el viejo agora con ele,

'Se fija bien el viejo ahora en ella

blanco como el mana espumas del mana olas,

'blanca como las espumas de las olas

como un plor del olas;

'como flor de las olas

el ojos negro, redondo como lomboy;

'los ojos negros, redondos como "lomboy"17

el labio presco, colorao como macopas;

"los labios frescos, colorados como "macopas"18

y el pelo negro, como el noche que tá rompé el luna agora

'y el pelo negro, como la noche que rompe la luna ahora...'

Después de comparar el uso de las partículas preverbales ta y $a$ con las partículas de los criollos filipinos ta y ya se puede contactar que estas se comportan tal como lo predice el prototipo de Bickerton, de manera que a pesar de que muchos de los rasgos del prototipo no se manifiestan en estos criollos, algunos otros en efecto se comportan de acuerdo con ese modelo. 


\section{Notas}

1. El lunfardo, tal como lo conozco, es más bien una jerga utilizada por las clases populares de Buenos Aires. Se caracteriza básicamente por tener un vocabulario especial y no manifiesta ninguna particularidad estrutural que muestre pidginización. Acerca del cocoliche, ver Whinnom (1971).

2. German de Granda se refiere obviamente a la forma española y no criolla de este pronombre, el cual se generalizó como bo en los criollos españoles.

3. Conservo en los ejemplos la grafía de los autores, por cierto, inconsistente.

4. Yang (1988: 39) agrega con respecto a la colocación de lo: "Lo se coloca antes del tema o los preverbos [vg. partículas preverbales] o combinaciones de ellos con que entra en secuencia (...), cuando el sujeto no es un pronombre personal. Si se añade la partícula negativa no, lo debe precederla."

5. Excepto cuando se indica otra cosa, los ejemplos son de ermitaño.

Del tagalo (Whinnom 1956: 29).

"The origins of this Ermitaño future tense or unreal aspect must lie in the Spanish (or Portuguese) haber de llorar. This new analytical future is extremely interesting, paralleling as it does the change from the synthetic Latin cantabo to the Vulgar Latin (and later Romance languages) cantare habeo, 'I have to sing', therefore 'I shall sing'. Spanish haber de cantar indicated originally obligation (...). In Spanish South American the form with haber has come to have no more than future force, and (...) has replaced the simple future in a great many instances" (Whinnom 1956: 34-5). Debe observarse que este futuro con haber de no es de ninguna manera de uso generalizado en Hispanoamérica, siendo la forma favorita para la referencia al futuro la construcción ir $a+\mathrm{V}$. Al mismo tiempo, el futuro simple tiene funciones muy restringidas, básicamente para la expresión del modo hipotético, como en Qué hora será; No sé si ellos irán a venir. En el español de Costa Rica la construcción haber de es muy restringida, y se usa probablemente solo para un modo hipotético particular, como en Ha de venir a pedirme un favor para que vea lo que le digo.

8. La siguiente oración en el texto es: polque ese gente de allí con nisós masiao malo el boca 'porque esa gente de allá, ellos hablan muy mal de nosotros.'

9. El verbo español parir nunca se usa en presente o habitual, (* yo paro ), sin embargo es muy frecuente en otros tiempos: parí, pariré, etc. Esto obviamente se debe a improbabilidad situacional: nadie tiende a estar hablando de parir en el momento de hacerlo.

10. No queda claro por qué los autores transcriben esta oración como vac' atá güeno, en lugar de vaca ta güeno.

11. Chuchubi es un pájaro (mocking bird).

12. Andersen indica un marcador 0 de subjunctivo en papiamento; no he considerado ese asunto en este trabajo, pero resulta interesante el hecho de que, como puede verse en el texto palenquero, el subjuntivo también puede analizarse como marcado por cero: güeno que y 0 pari un moná '(es) bueno que yo tenga un hijo'.

Información suministrada por Assane Diagne, de Dakar, Senegal.

$m a$ 'yo' se convierte en na en pasado progresivo y en futuro.

Acerca de esta expresión, Whinnom (ibid: 38) señala: “One is reminded of Sp. nada más (...). But this leads us into difficulties, since the translation of no namás is 'no less than'. It is conceivable that the na is the Tagalog na 'already', but this would give us 'no more than' instead of 'no less than'. Perhaps no nada mas que = algo más que "rather more than', in which case na would be a contraction of nada." 
17. lomboy: del tagalo, una especie de mora (blackberry, Whinnom ibid: 39 ).

18. macopas: "The word is not Tagalog, Spanish, nor Portuguese. I have no explanation of it." (Whinnom ibid: 39$)$.

\section{Bibliografía}

Andersen, Roger W. 1990. "Papiamentu Tense-Aspect, With Special Attention to Discourse". En: John V. Singler (ed.).

Bickerton, Derek. 1974. "Creolization, Linguistic Universals, Natural Semantax and the Brain". En: Richard R. Day (ed.), 1-8.

1979. “Beginnings”. En: Kenneth C. Hill (ed.).

Bickerton, Derek y Aquilas Escalante. 1970. "Palenquero: A Spanish-Based Creole of Northern Colombia”. Lingua , 24: 254-67.

Crystal, David. 1987. The Cambridge Encyclopedia Of Language. Cambridge: Cambridge University Press.

Ferrol, Orlando. 1982. La cuestión del origen y de la formación del papiamento. La Haya: Smits Drukkers-Uitgevers.

Givón, Talmy. 1984. Syntax. A Functional-Typological Introduction. Vol I, Amsterdam: John Benjamins.

Granda, German De. 1968a. "Sobre el Estudio de las Hablas Criollas en el Area Hispánica”. Thesaurus, Boletín del Instituto Caro y Cuervo. Vol. XXIII.

1968b. "La Tipología 'Criolla' de Dos Hablas del Area Lingüística Hispánica”. Thesaurus, Boletín del Instituto Caro y Cuervo. Vol. XXIII.

1978. Estudios lingüísticos hispánicos, afrohispánicos y criollos. Madrid: Gredos.

Hesseling, Dirk C. 1979. "Papiamentu and Negerhollands". En: On The Origin And Formation Of Creoles: A Miscellany Of Articles. Ann Arbor: Karoma Publishers.

Holm, John A. 1988. Pidgins And Creoles. Vol I. Cambridge: Cambridge University Press.

Lipski, John M. 1984. "Observations on the Spanish of Malabo, Equatorial Guinea: Implications for Latin American Spanish”. Hispanic Linguistics. 1: 69-96. 
1985. "Creole Spanish and Vestigial Spanish: Evolutionary Parallels". Linguistics. 23: 963-84.

1987. "The Chota Valley: Afro-Hispanic Language in Highland Ecuador". Latin American Research Review. 22: 155-70.

1989. The Speech Of The Negros Congos Of Panama. Amsterdam: John Benjamins.

Singler, John V. 1990. Pidgin And Creole Tense-Mood-Aspect Systems. Amsterdam: John Benjamins.

Whinnom, Keith. 1956. Spanish Contact Vernaculars In The Philippine Islands. Hong Kong: Hong Kong University Press.

Yang, Mei-Ling. 1988. El sistema preverbal del papiamento y la hipótesis innatista de D. Bickerton sobre el origen de los indicadores de tiempo y aspecto en las lenguas criollas. Tesis de Maestría: Universidad de Costa Rica. 
